\title{
Las características de la situación mental en el consumo adictivo de sustancias y la motivación para entrar a un tratamiento de adicciones
}

\author{
Characteristics of the mental situation involved in the \\ addictive consumption of drugs and the motivation to \\ enter an addiction treatment
}

Cristián López Acosta

\section{Resumen}

En este artículo se presentan los resultados de un estudio acerca de las características de la situación mental de sujetos que han desarrollado una adicción, en una etapa previa a la decisión de consultar. Esta situación psíquica es relacionada con la dificultad en el proceso de toma de decisión de entrar a tratamiento, propia de estos pacientes. A partir de estos resultados se discute el concepto de motivación a tratamiento y los elementos técnicos asociados a este. También se discute la relevancia de incluir a otros significativos y la importancia de lograr una detención inicial del consumo de drogas.

Palabras clave: abstinencia, adicción, motivación, psicoanálisis, tratamiento.

\section{Abstract}

This article contains the results of a research regarding the characteristics of the mental situation of individuals that have developed an addiction, during the stage previous to seek advice. This psychic situation is related to the difficulty of the decision process involved in entering a treatment, typical in these patients. As from the results, a discussion arises entailing the concept of motivation in

1 Académico Escuela de Psicología Universidad Católica de Chile. Doctor en Psicología Universidad de Chile. Santiago, Chile. E- mail: cristian@uc.cl 
relation to a treatment and the technical elements that are associated thereof. It is discussed also, the relevance of including significant others, and the importance of achieving an initial suspension of the drugs consumption.

Key words: addiction, abstinence, motivation, psychoanalysis, treatment.

\section{Introducción}

Durante el siglo XX, hasta la década del setenta, los tratamientos dirigidos a sujetos que habían desarrollado una adicción tendieron a no abordar el tema de la motivación a tratamiento. La entrada a tratamiento se daba en el contexto de un sujeto dispuesto voluntariamente a recibir ayuda o, menos habitualmente, en un contexto de obligatoriedad, ya fuera por razones judiciales o médicas. Hasta finales de la década del sesenta casi todos los programas de tratamiento de EEUU utilizaban la aproximación de los doce pasos de Alcohólicos Anónimos (AA), e incluso a comienzos de la década del noventa, en el sector privado, el 93\% de los programas de tratamiento de ese país seguía basado en el mismo modelo (Peele, 1998, citado en Sobell \& Sobell, 2006). La perspectiva de AA, así como de los tratamientos de autoayuda, en general, trabaja con sujetos que desean tratarse (Alcohólicos Anónimos, 1995). De esta manera, la medicina en conjunto con los grupos de autoayuda fue una alternativa asequible solo para sujetos que ya tenían una motivación para enfrentar el problema.

Desde la perspectiva psicoanalítica, por su parte, se pueden distinguir dos grandes aproximaciones a la entrada a tratamiento de sujetos que han desarrollado una adicción. Una de ellas incluye autores que reconocen que este tipo de pacientes requiere eventualmente un abordaje distinto del tradicional, incluyendo en la propuesta terapéutica intervenciones como la hospitalización, la asistencia a una comunidad terapéutica o al hospital de día, y la petición de abstinencia (Ghia, 2000; Ingelmo, Ramos, Mendez \& González, 2000; Kameniecki, 2000; Kielholz, 1924, Knight, 1937, 1938, Merloo, 1952, Weijl, 1928, citados en Rosenfeld, 1964; Sillitti, 2000a; Tarrab, 2000b). Desde la segunda 
aproximación, se ha criticado esta idea de combinación de intervenciones terapéuticas, ya que respondería a una oscilación entre una posición médica y una posición psicoanalítica, perdiéndose la lógica que guía la cura. Los autores que sostienen esta perspectiva, a pesar de estar de acuerdo en que la entrada en un proceso terapéutico de este tipo de pacientes es especialmente dificultosa, tienden a proponer la aplicación de una lógica de la cura sin modificaciones esenciales, que apunte a la emergencia del sujeto del inconsciente, suponiendo que a la base de la conducta adictiva habrían mecanismos o estructuras psíquicas propias del sujeto que tendrían que ser modificadas para que se generara un cambio en la conducta adictiva (Luongo, 1995; Mazzini, 1994; Salamone \& Godoy, 1994; Salamone, 1995; Sillitti, 2000b, 2000c; Sinatra, 2000a, 2000b; Tarrab, 1994, 2000a). Ambas aproximaciones psicoanalíticas soslayan, al igual que la perspectiva médica-grupos de autoayuda, la posibilidad de realizar intervenciones dirigidas específicamente al problema de la motivación a tratarse.

A partir de la década del setenta, en contextos teóricos y técnicos muy diversos, surgen intentos de abordar explícitamente esta problemática, hasta convertirla en un tema principal de investigación hoy en día. Esta atención se ha generado debido a que solo un porcentaje minoritario de la población con problemas de abuso o dependencia consulta en algún momento de su vida (Cunningham \& Breslin, 2004), y a que un porcentaje importante de este grupo que consulta, lo hace a instancia de terceros significativos (Gerdner \& Holmberg, 2000, Gregoire \& Burke, 2004, Joe et al., 1999, Polcin \& Weisner, 1999, Rush \& Wild, 2003, Weisner, 1990, citados en Wild, Cunningham \& Ryan, 2006). Inicialmente, la investigación estuvo dirigida a desarrollar intervenciones que llevaran a que el sujeto aceptara un tratamiento, para luego dirigirse al desarrollo de intervenciones que buscaran la instalación de una motivación propiamente tal. En términos generales se podrían distinguir las intervenciones de aceptación-motivacionales que colocan el énfasis en el trabajo con terceros significativos (Berenson, 1976; Johnson, 1973; Landau et al., 2004; Santisteban, Suárez-Morales, Robbins \& Szapocznik, 2006; Szapocznik \& Williams, 2000; Thomas, Adams, Yoshioka \& Ager, 1990), y las intervenciones que colocan el énfasis en el trabajo individual (Beck, 
Wright, Newmann \& Liese, 1999; Miller \& Rollnick, 1999; Prochaska \& DiClemente, 1984; Janis \& Mann, 1977, citados en Share, McCrady \& Epstein, 2004). También se han desarrollado modelos de intervención que intentan integrar la dimensión personal e interpersonal al momento de fomentar la entrada a tratamiento (Azrin, 1976; Kreither \& López, 2001; López, 2006; Miller \& Meyers, 1999).

Estas intervenciones motivacionales, en general, están enmarcadas en una noción de tratamiento que supone una detención o una disminución del consumo de sustancias adictivas como objetivo. Varían en términos de cómo entienden el obstáculo psíquico que impide que la persona se trate, y la medida en que involucran la participación y/o influencia por parte de terceros para que el sujeto acepte ayuda. La forma más tradicional de concebir este obstáculo psíquico está dada por la idea de la negación como un mecanismo que permite al sujeto no dimensionar las consecuencias en la realidad de su conducta adictiva. Desde una perspectiva psicoanalítica, esta negación ha sido entendida como un mecanismo de defensa propio de la estructura psíquica del sujeto (Miller \& Rollnick, 1999). Desde el psicoanálisis también se han definido otras condiciones psíquicas propias del sujeto en consumo adictivo. Aunque no están planteadas desde la perspectiva de la motivación, desde los enfoques motivacionales estas condiciones se podrían entender funcionando como obstáculos para una decisión de tratarse. Entre ellas se puede nombrar las fuerzas libidinales separadas del yo (Sachs, 1923), la instalación de un deseo morboso (Rado, 1926) y un régimen farmacotímico del yo (Rado, 1933), la escisión del self (Rosenfeld, 1964), la escisión de una imagen de si mismo (McDougall, 1994) o la multiplicidad de estados del self (Burton, 2005). La noción de Rado de la instalación de un régimen farmacotímico es especialmente interesante, ya que no hace alusión a mecanismos o estructuras existentes previamente al desarrollo de la adicción, idea que sí caracteriza a los entendimientos psicoanalíticos hasta el día de hoy, sino que a un modo de funcionamiento psíquico propio de la situación mental del sujeto adicto. La noción de situación mental, en este artículo, aludirá a un estado mental coyuntural al estar inmerso en un consumo adictivo, en oposición a una estructura psíquica estable del sujeto existente más allá de su conducta de consumo adictivo. 
Desde una perspectiva médica, por su parte, la negación se concibe como una distorsión del pensamiento tal como aparece en la conocida definición realizada en 1992 por el Joint Committee of the National Council on Alcoholism and Drug Dependence and the American Society of Addiction Medicine (Morse \& Flavin, 1992). La idea de mecanismos de defensa a ser atravesados, o la de distorsiones a ser corregidas, favorece el desarrollo de métodos confrontativos para intentar superarlos. Una alternativa a este entendimiento tradicional del problema, realizado desde la medicina, desde los grupos de autoayuda, y desde los autores psicoanalíticos que piensan que hay que hacer algo específico en este tipo de casos, se puede encontrar en las propuestas derivadas de la terapia cognitiva (Beck et al., 1999), en los modelos de las etapas de cambio de Prochaska y DiClemente (1984), y especialmente en la propuesta de la entrevista motivacional de Miller y Rollnick (1999). A partir de estos modelos, el énfasis ya no queda puesto en mecanismos de defensa propios del sujeto que operan como resistencia, o en una distorsión del pensamiento. En estos modelos se considera que, como parte de un proceso normal y no patológico, el sujeto tiene motivaciones fluctuantes y conflictivas, que lo llevan a querer pero no querer hacer algo con su conducta adictiva, fenómeno al que llaman ambivalencia. La labor terapéutica, entonces, no consiste en confrontar al sujeto con los mecanismos patológicos que le impiden cambiar, sino en permitirle desplegar la ambivalencia confiando que ya existe la disponibilidad suficiente en el sujeto para el cambio. En la medida que esa ambivalencia se pueda desplegar, reconocer y analizar, el sujeto irá pasando las sucesivas etapas que lo harán tomar decisiones de cambio en una dirección positiva, entendida, o más bien sobreentendida, como una detención o disminución del consumo. Sin embargo, es cuestionable que el concepto de ambivalencia desarrollado por estos enfoques pueda dar suficiente cuenta de la situación mental que caracteriza al sujeto en consumo adictivo.

La situación mental en la que se encuentra el sujeto inmerso en un consumo adictivo de sustancias antes de consultar, requiere ser investigada con mayor detalle, con el fin de aportar en la discusión sobre nociones como motivación, ambivalencia, defensas, y en la consiguiente discusión 
sobre los modos de abordaje terapéuticos. Esta situación mental parece tener características particulares, que no dejan identificarse y precisarse adecuada y suficientemente cuando son abordadas desde nociones desarrolladas para dar cuenta de otro tipo de fenómenos. Con el fin de contribuir a identificar y comprender estas características de la situación mental del sujeto, se presentan los siguientes resultados obtenidos en un estudio realizado con sujetos que participaron de un proceso de entrada a un tratamiento de adicciones. Será de relevancia discutir la relación entre estas características, el proceso de toma de decisión de realizar un cambio en el consumo adictivo por parte del sujeto, y la pertinencia de algunos elementos para una intervención terapéutica. Los resultados mostrados en este artículo son complementarios a otros, producto del mismo estudio y previamente publicados, referidos a la influencia de terceros en la motivación a tratarse (López, 2009).

\section{Metodología}

\section{Participantes}

Los sujetos seleccionados provienen de un grupo de pacientes con diagnóstico de adicción a sustancias químicas que participaron de una intervención motivacional inicial llamada entrevistas previas (Kreither \& López, 2001) y luego continuaron en un programa de tratamiento. Al momento de la entrevista, cinco de los seis pacientes ya habían culminado el proceso de tratamiento, y uno de ellos aún estaba participando de él. Cinco de ellos consultaron por consumo de alcohol, marihuana y cocaína, y uno solamente por alcohol. Uno de los seis pacientes pasó por una etapa inicial de hospitalización. Las edades fluctúan entre los 26 y 40 años, cuatro de ellos estaban casados. Al momento de realizarse la entrevista los pacientes ya habían pasado al menos hace nueve meses por el proceso de entrevistas previas. Se escogió de esta forma a los sujetos con el objetivo que no estuvieran interferidos por el vínculo adictivo con la sustancia en su percepción de lo vivido. 
Con cada uno de los sujetos se realizó una entrevista en profundidad. Esta duró entre una y una hora y media. En esta entrevista se les pidió a los sujetos que relataran libremente los recuerdos que tenían de su experiencia en relación a las primeras semanas de su llegada a consultar. Esta técnica se escogió debido a que es un instrumento que permite el libre despliegue de un relato acerca de las vivencias del sujeto. Aporta una información que será entregada con una orientación e interpretación significativa sobre lo vivido, que es lo que se busca relevar en oposición a una recolección de hechos (Alonso, 1995; García Ferrando, Ibañez \& Alvira, 1989). Para los efectos de este estudio interesaron en particular dos campos de la entrevista en profundidad: el campo referido a la reconstrucción de acciones pasadas, específicamente el análisis retrospectivo de la acción, y el campo del estudio de representaciones sociales personalizadas.

\section{Análisis de datos}

Los resultados fueron elaborados sin seguir un procedimiento algoritmizado llevado a cabo según reglas a priori que indicaran cómo proceder. En contraposición a esto, se utilizó la línea de análisis de contenido de Ibáñez (1979), que valida la intuición, experiencia y conocimiento teórico como herramientas de lectura de los datos. Este método busca permitir una interpretación y organización de los datos, sin perder el ámbito de la subjetividad desde donde se habla, obligando a una constante vigilancia epistemológica que analice las condiciones que mueven a interpretar como se hace. Se crearon categorías inductivamente, que permitieron vaciar los contenidos de las entrevistas, distinguiéndose elementos comunes y particulares. También se distinguieron distintos momentos en el tiempo del proceso de decisión. Para finalizar se elaboró un mapa de las posiciones discursivas de los sujetos. 


\section{Resultados}

En los resultados del análisis de las entrevistas se distinguen cuatro momentos relevantes del proceso de decisión de entrar a un tratamiento: (1) La situación mental del sujeto previa a la llegada a consultar; (2) la decisión de entrar a un período de evaluación (o etapa motivacional inicial); (3) el momento de la "entrada"; (4) el período de evaluación.

Para los efectos de este artículo solo interesará el punto (1) de los resultados, el que, a su vez, está compuesto de los dos siguientes subpuntos: (i) la desorganización subjetiva y (ii) la oscilación de las posiciones subjetivas. A continuación se describen los resultados asociados a estos sub-puntos.

\section{La desorganización subjetiva}

Todos los pacientes entrevistados describen una situación mental previa a su llegada a consultar, marcada por una fuerte sensación de desorganización en su forma de funcionamiento. Existiría una percepción de vivir establemente en una posición crítica, a la cual no se le ha podido encontrar una salida. A pesar de esta desorganización en el funcionamiento, se mantiene la capacidad de realizar las actividades necesarias para conseguir las sustancias y poder consumirlas. Se pueden distinguir varios aspectos relacionados con este punto.

\section{La pérdida de si mismo y la dificultad en la relación con la realidad}

Algunos pacientes tienen la impresión que esta desorganización afecta solo a algunos aspectos de su vida, y otros la viven como algo que cruza todos los ámbitos. La desorganización se puede vivir como una falta de capacidad y/o motivación en la realización de las actividades que no tienen que ver con el consumo de alcohol:

- "No rindes en la pega, lo que tú quisieras rendir, no rindes en la casa, lo que quisieras rendir, no rindes lo que tú crees que eres capaz de rendir, por lo tanto algo pasa, que yo ando impávido por la vida, lo 
que me digan da lo mismo mientras... pase piola, ¿qué? ¿ah?, total en el tumulto paso colado".

También puede ser vivida como una sensación de haber perdido algo propio de sí mismo:

- "Si uno no es sujeto".

- "Cada vez que yo me tiraba algo es como que me pasaba a llevar, era como que me trataba de parar, me tiraba algo... y al suelo, al suelo, todo el rato al suelo, era como que me pasaba llevar a mi mismo, todo el rato".

El dejar de ser "sujeto" habla de una pérdida profunda de la capacidad de tener una posición organizada frente al medio. Otra forma de experimentar esta pérdida sería a través de la impresión de una profunda transformación en sí mismo:

- "Me estaba transformando en un monstruo".

Esta transformación de sí mismo se ve también como un haber dado un paso hacia la locura, hacia la pérdida del sentido de las cosas:

- "Y de hecho, después ya con la lucidez y con el tiempo me di cuenta que era un huevón loco, no es que yo haya estado, era, eso me tenía muy angustiado".

- "También me daba cuenta de los rayones que me pegaba, paranoia, claro, veía cómo estaba mi vida, tenía la cagada en mi vida, la embarrada gigante que tenía por todos lados, porque habia quemado todas las alternativas".

La desorganización subjetiva va produciendo una relación muy conflictiva con la realidad en sus distintos planos, lo que puede llevar a que esta realidad comience a ser percibida como inmanejable a pesar de no estar viviendo necesariamente circunstancias objetivas catastróficas:

- "Ya estaba completamente superado por la vida que estaba viviendo".

- "El día a día es muy malo eh... todas las... desorden alimenticio, el no poder mirar a la cara".

La desorganización también puede percibirse como afectando las capacidades cognitivas:

- "Yo ya me daba cuenta que... de que no podía hilar bien las ideas". 


\section{La idea de la muerte y el suicidio}

Esta desorganización del sujeto en ocasiones lleva a vivir en situaciones límite, lo que puede asociarse con la aparición de ideas suicidas claras y recurrentes, que parecen tener el sentido de encontrar finalmente una salida a lo que se está viviendo:

- "Sí, de hecho, no sé, mi viejo tenía una pistola en la casa, súper fácil, o conseguirse una pistola, súper fácil, donde andaba metido era fácil... es rápido, no tan doloroso, y de hecho fantaseaba con la cuestión, y me tranquilizaba, me venía la idea que mañana se acababa".

- "Yo pensé quitarme la vida [...] pero era una idea... una idea, una salida más, porque cómo enfrentaba yo ese escenario, ya no podía enfrentarlo [...] en un momento 'qué, me pego un tiro"'.

El fantasear con el suicidio incluso puede llevar a que el sujeto sienta alivio frente a lo que está viviendo, lo que deja ver lo angustiante e insostenible de la sensación de desorganización en la que se encuentra.

La muerte aparece en el horizonte de los sujetos no solo con la noción de suicidio. También se hace presente a partir de la impresión de catástrofe inminente en la cual se puede llegar a vivir. Esto puede causar la emergencia de un fuerte temor por las cosas que pudieran suceder producto del estado mental en el cual el sujeto se encuentra:

- "Eso me tenía muy atemorizado, porque cada vez que consumía estaba perdiendo la conciencia, y eso me daba mucho miedo, por eso estaba condenado a muerte [...] yo estaba condenado a muerte, o yo me iba a morir o me iban a matar, yo vivo no iba a estar mucho tiempo".

- "Y el haber chocado tantos autos, o sea, por decirte algo, el haber estado tan cerca de matarme tantas veces".

- "Entonces, era un espiral de violencia que se venía [...] que ya estaba perdiendo absolutamente el control, me estaba metiendo en el tráfico, estaba jugando con fuego, y de ahi no iba a salir vivo, de ninguna forma".

El matar a otros también es algo que está dentro de las alternativas que se saben posibles: 
- "Pero ahi dije 'puedo terminar en una grande cachai, o sea, hacer algo realmente grande, como matar a alguien, que realmente no tuviera una vuelta tan fácil, como que habia perdido la... de hecho ya no me importaba nada, era tal cual, me asustaba de volverme eso, pero habia perdido la importancia por las cosas, todo, todo".

- "O yo iba a matar a un huevón porque lo atropellé curado".

Las conductas antisociales y los sentimientos de culpabilidad

Otro aspecto que aparece asociado a esta desorganización es la aparición de un cambio en la forma de relacionarse con los demás, que puede dar pie a conductas antisociales en las cuales el sujeto no se reconoce:

- "El haberme cagado a tanta gente [...] las relaciones que mantenía con toda la gente, o sea, no eran relaciones, o sea, yo miraba si podía tener algún provecho, y según eso lo tomaba o lo descartaba, cero sentimiento, cero conexión con la gente, era provecho o no provecho, me servía o no me servía".

También hay sentimientos de culpabilidad importantes, asociados al daño que se habría producido a otros significativos, que forman parte del malestar del sujeto:

- "Y súmale la carga si es que tenís un hijo... eso a mi me pesaba harto, verdad [...] para mi el tema de P. [hijo], hablado asi en las sesiones, era un tema que me dolía mucho... me sentía como muy culpable, muy mala persona, mal padre".

- "Ya no me importaba la gente [... J ya está bien lo que hago yo cachai, pero cuando empiezo a tocarte a ti y a no respetarte a ti, eso me empezó a asustar un poco, ya estaba haciendo cosas que pasaba el limite de lo antisocial, como cagarte, si te podia cagar te cagaba, cachai, como estar conversando contigo, pretender que me importai, pero a la primera que te podia cagar te cagaba". 
La Oscilación de las Posiciones Subjetivas como un Elemento Central de la Situación Mental

Un elemento que parece ser de gran importancia en la situación de "desorganización subjetiva" inicial es el fenómeno de coexistencia de dos posiciones del sujeto contradictorias y acompañadas de la cualidad de conciencia. Esta oscilación es un factor muy importante al momento de ser capaz de tomar decisiones.

El sujeto sabe que "está mal" y que debe hacer algo con su consumo

Por una parte, el sujeto sabe que "está mal", asociando muchas veces este estar mal con el consumo de sustancias:

- “'Hay algo aqui que no está cuajando, hay algo aqui que’ [...] y entonces cuando llegaba a la casa doblado, con ella, de algún carrete, y al día siguiente no me hablaba [la esposa], y yo decía estoy cagado, nunca más, estaba bien dos, tres semanas, a la otra semana... otra vez... entonces tú decís... como que no cuaja".

- "Ya tenía conciencia, una cierta conciencia, de que estaba mal, de que estaba en algo mal, que mi vida estaba totalmente mal, angustia también que se fue desarrollando en forma ascendente".

- "Si, si... yo lo tenía súper claro, yo de hecho sentía que tenía un alcoholismo asi fuerte, digamos".

- "Yo sabia que estaba metido en algo complicado, sabia que no iba a poder salir solo y tenía la esperanza de poder tener una vida distinta”.

- "A lo que te lleva todo este consumo en los carretes, que siempre es medio sórdido al final de toda la cuestión y de repente llega un punto en que decís 'estoy mal'".

Muchas veces, además de saberse que se está mal, se sabe que es necesario hacer algo en relación a la situación que se está viviendo:

- "Sabes que tienes que hacer algo. No sabes qué. Estás metido en el cuento, el elástico lo estiras hasta que se corta".

- "Entonces, por no saber, y ya sentís que la huevada no calza, entonces decís 'hay que hacer algo'”. 
- "Me recuerdo cientos de noches en que yo decía 'tengo que salir de esta cuestión, estoy mal', entonces, y esos minutos eran de angustia terrible, porque lo unico que querís es quedarte dormido".

La oscilación subjetiva y la paralización de la capacidad de tomar decisiones

Esta situación es particular, ya que a pesar de saber que se "está mal", y saber que es necesario "hacer algo" por salir de la situación insostenible, no es posible tomar la iniciativa y realizar acciones que puedan concretar esta intención. Se sabe que la solución pasa por modificar el consumo de sustancias y se tiene la intención de disminuir o detener este consumo. Pero, por otra parte, llegado el momento todo se "olvida" y se vuelve a consumir las sustancias como si no hubiera habido ninguna reflexión al respecto y como si el aspecto de la realidad que se veía desde la primera posición cambiara radicalmente:

- "Tratai por las tuyas, y no podís, y de repente podía aguantar más tiempo y las recaidas son mucho más fuertes poh, entonces yo sabia que no podía tomar alcoholes destilados, entonces agarraba vuelo con unos compadres, nos íbamos para arriba y... métale piscolas... sabíai que no podiai, estabai consciente que no podíai... igual no más".

- "Desde hace mucho tiempo, desde el momento en que empieza a ser algo progresivo, en que las cosas se hacen más frecuentes, ya no tan a lo lejos... ahi ya uno, uno se empieza como a cuestionar un poco el... realmente si tiene el control o no [...] entonces uno empieza como intimamente a pensar si lo tiene controlado o no, y, claro, se miente un poco, te decis 'a ver, este fin de semana voy a tratar de no'... tratar de mostrarle a los demás un poco, cosa que normalmente termina no funcionando".

- "O sea, imaginate pasar horas pensando, después de haberme drogado todo el dia, eh... yo ya no quiero seguir asi', pero teniendo conciencia de que a la mañana siguiente iba a estar de nuevo en la misma, porque no tenía nada, ni la voluntad, nada". 
Se produce una oscilación entre dos lugares desde los cuales se toman decisiones, ubicación del sujeto que puede variar en forma rápida dependiendo de las circunstancias en las cuales se encuentra. Esta oscilación de la ubicación llevaría a no poder sostener las determinaciones que se han tomado en relación a hacer algo con la situación que se está viviendo, en particular con el consumo de sustancias:

- "Yo, en el fondo, ya tenía absoluta conciencia que tenía que hacer un cambio, me costaba, no podia hacerlo porque estaba metido hasta las patas".

- "Yo eh... tenía ganas de contarlo todo [...] o sea, por una parte, necesitaba ese espacio, pero, por otra parte, también yo veía que eso iba a traer consecuencias y que se me iba a caer todo el mundo encima".

- "Hay una cosa de cómo uno lo siente, de antes... uno ya tiene la sensación. Cada vez que uno se manda una cagada, una cosa así, le viene un como 'irealmente estaré mal?, jestará realmente incontrolable esta situación?'... cosa que uno pensaba, pero quedaba ahi no más, o sea después se perdía".

El sujeto queda ubicado en dos lugares contradictorios que parecen no influirse uno a otro, vive con la impresión de estar radicalmente dividido:

- "Lo que pasa es que esto es como... Dr. Jeckyll y Mr. Hyde, es así, entonces cuando tú eres Dr. Jeckyll tienes la lucidez como para darte cuenta que Mr. Hyde es un enfermo, y lo más curioso es que eres la misma persona. Y eso te provoca miedo. Miedo el pensar el monstruo que tengo adentro".

- "Era decir 'puta gracias, sáquenme por favor', cachai, es verdad, me estaba transformando en una persona muy peligrosa, y eso era... era triste... Pensaba y a veces miraba fotos de cuando yo era pendejo y a ratos pensaba qué lata que este niño... se transformó en un monstruo. $Y$ es verdad, asi me sentía".

- "Durante todo el periodo de consumo, eh... uno es dos, verdad, y en mi caso con mayor razón, porque mi consumo era un consumo escondido, entonces era un dos que solamente conocía yo, un dos que vivía gran 
parte del día, quizás más que el otro... te fijai, era este que preocupado de dónde sacaba la plata, preocupado de ir a comprar, preocupado de buscar el minuto, eh... volao, el bajón. Entonces, era un dos que vivía conmigo quizás más parte del tiempo de este otro que vivía con el resto de la gente... Entonces esta dualidad te desgasta un montón".

- "Pero en el momento no estaba ni ahi, pero al otro día... y de tanta acumulación de eso me decía loco...', pero no, no habia real conciencia de esto, en el momento no, pero cuando me veia, no me calzaba, y eso me generaba... O sea, si yo lo hubiera encontrado bien, está todo bien, no hay ningún problema, pero como te digo, no me calzaba, para nada, no era lo que yo era, o lo que yo tenía idea de lo que era".

La influencia del consumo adictivo en la oscilación subjetiva

La relación adictiva con las sustancias, el deseo por ellas y su consumo, influye fuertemente sobre este girar de la perspectiva, que lleva al no poder fijar una posición frente a un tema que está teniendo grandes consecuencias, y a realizar las acciones para encontrar una salida a la situación:

- "Sí, varias veces, por ejemplo, me propuse, 'ya, este fin de semana no voy a consumir cocaina'.. Ib a a una fiesta, me ponia a tomar, y bastaba que alguien me ofreciera y hasta ahi llegaba la promesa. O sea, no tenía mucha voluntad $y . .$. y con los pitos, si trataba de no fumar un dia, por ejemplo, pero más que eso no podia".

- "Ahora, cuando ya estaba metido en el tema de la coca, yo la verdad no sabía cómo iba a terminar, porque era tal el vicio que los pensamientos fugaces de querer emprender una acción para salir, eran milisegundos en relación a los deseos de consumo y rápidamente se me pasaban, porque quería ir a consumir".

- "Yo, en realidad, cientos de noches pensaba que estaba pa' la cagá y que tenía que cambiar, que dejar el consumo. Lo que pasaba es que a la mañana ya eso se me habia olvidado, y lo único que pensaba era en ir a comprar". 
- "La forma en que me conducía, el arrepentimiento del otro día, era como que en un momento estaba muy muy consciente de un problema y, en un segundo momento, más tarde, era, 'ya filo, no importa, vamos'. Aparecía toda la mecánica de nuevo de conseguirme lo que tenía que conseguirme, y cuando me lo estaba tirando, 'está todo bien, qué bueno".

\section{Discusión}

Los hallazgos de este estudio provienen de una visión en retrospectiva, con al menos nueve meses de distancia, de sujetos que participaron de una intervención motivacional previa a un tratamiento de adicciones. Por una parte, esta distancia temporal favorece que el recuerdo de la vivencia no esté interferido por un vínculo adictivo actual con la sustancia química. Ese vínculo adictivo haría entrar en juego los intereses psíquicos propios de un deseo de consumo que inclinarían los recuerdos en una dirección determinada. Por otra parte, el tiempo transcurrido puede tergiversar el recuerdo de la vivencia, ya que sería esperable que este estuviera influido por toda la elaboración posterior que el paciente ha ido haciendo en torno al tema. Esta es una limitación del estudio que es necesario tener en cuenta.

Los resultados presentados son producto de un vaciado de los contenidos de los seis sujetos participantes en categorías inductivamente elaboradas. Esto hace que se pierda la posibilidad de situar la posición subjetiva de cada sujeto particular en relación a la posibilidad de una entrada a tratamiento. En ese sentido, este estudio se aleja de lo que sería un análisis de caso que busca enfatizar la particularidad de cada sujeto. En lugar de esto, se intenta encontrar lo común en el relato de los seis sujetos en relación a su situación mental antes de producirse la consulta. Esta forma de realizar el análisis lleva a que se pierda el contexto específico de cada sujeto, que podría enriquecer y ampliar el entendimiento de la situación particular. Por otro lado, favorece la posibilidad de encontrar elementos generalizables a otros sujetos.

En los resultados se encuentran dos características principales, que pueden considerarse propias de la situación mental del sujeto. La primera característica es la desorganización subjetiva. Esta aparece relacionada con la presencia de ideas de muerte y suicidio, estados depresivos y 
angustiosos, serias dificultades en la relación con la realidad, problemas cognitivos y conductas antisociales. El sujeto siente que ha perdido en medida importante su horizonte y proyecto de vida, pudiendo llegar a vivirse como completamente anulado, al punto de llegar a desconocerse. Esta desorganización se traspasa a su situación de vida, favoreciendo que el sujeto llegue a tener conductas que colindan con lo antisocial.

Esta primera característica de la situación mental, por una parte, favorece que en el sujeto aparezca la vivencia de una necesidad de realizar un cambio importante en su vida, de forma de dejar de vivir al límite o en crisis crónicas. Los sujetos relatan haber tenido una clara conciencia de que las consecuencias de su conducta de consumo adictivo eran insostenibles. No aparece una negación de lo que estaba ocurriendo, sino que, al contrario, una desesperación y angustia con la realidad que enfrentaban, acompañadas de una sensación de derrota por no poder hacer algo al respecto. No hay una falta de motivación, debido a un no ver la realidad. Sin embargo, por otra parte, esta desorganización subjetiva dificulta la capacidad de organizar una respuesta que permita cambiar la situación. Cambiar esta situación implica tomar decisiones que tendrán una serie de consecuencias muy importantes sobre su vida, no logrando el sujeto realizar un análisis racional y de conjunto de esas consecuencias ni de las alternativas concretas a seguir para realizar los cambios necesarios. Se tiene, entonces, a un sujeto que ya está fuertemente motivado a realizar un cambio significativo en su relación con las sustancias químicas, pero sin la capacidad de organizar esa empresa. En el relato de los sujetos, esta desorganización subjetiva aparece asociada al efecto intoxicante de las sustancias sobre lo anímico y lo cognitivo, a la angustia propia de las situaciones de vida críticas en las que vive inmerso, y a la lucha con un deseo de consumo siempre amenazante y potencialmente perturbador de cualquier intento de reorganización. Es decir, aparece relacionada principalmente con elementos coyunturales al consumo y sus efectos.

El análisis de esta primera característica lleva a cuestionar el énfasis colocado por el psicoanálisis y la perspectiva médica en mecanismos de defensa o distorsiones que impedirían al sujeto ver la realidad que está vivien- 
do. Corrobora, más bien, el entendimiento realizado desde los modelos motivacionales revisados, que suponen que el sujeto sí quiere hacer un cambio. Sin embargo, más que una ambivalencia, en el sentido de motivaciones fluctuantes, lo que aparece es una oposición entre una motivación a hacer un cambio y la aparición de un fuerte deseo, el deseo morboso postulado por Rado (1926), por consumir la sustancia. Este segundo elemento no parece formar parte de una estructura motivacional del sujeto, sino ser una irrupción en ese sistema que, dada la desorganización subjetiva posibilita olvidarse de todo y volver a consumir, echando por la borda cualquier intención que se haya tenido de organizar las acciones necesarias para llevar a cabo un cambio. La desorganización subjetiva es el terreno que permite que esa irrupción no pueda ser contrarrestada desde la motivación ya existente en el sujeto.

La segunda característica de esta situación mental es la de la oscilación subjetiva. Esta consiste en que el sujeto no es capaz de tener un visión unitaria de lo que está sucediendo con él. Es decir, reunir en un solo vistazo el que su situación mental y de vida requiere un cambio significativo y urgente en relación al consumo adictivo de sustancias; y el que su desorganización es tal que, frente a los deseos de consumo, no podrá sostener su motivación al cambio y, llegado el momento, decidirá olvidarse de todo y volver a consumir. El sujeto oscila entre una posición y otra, frecuente y permanentemente, quedando paralizadas sus intenciones de cambio en un ir y venir desgastante entre ambas posiciones. Esta oscilación subjetiva parece dar cuenta de la existencia de un profundo quiebre que compromete la sensación de identidad y el sentimiento de sí del sujeto. En este sentido, esta característica de la situación mental, característica que siguiendo los relatos transcritos podría llamarse Dr. Jeckyll y Mr. Hyde, parece acercarse a las nociones de algunos autores psicoanalíticos revisados, como Sachs (1923), Rado (1926, 1933), Rosenfeld (1960), McDougall (1994) y Burton (2005), que apuntan a una división psíquica radical en estos sujetos. Sin embargo, en el relato de los sujetos, este quiebre se muestra asociado con una situación mental propia del consumo adictivo de sustancias y no con una estructura psíquica previa propia del sujeto. 
Esta oscilación subjetiva produce una paralización en la capacidad del sujeto de llevar a cabo las decisiones que pueda haber tomado. Si, por el contrario, el sujeto fuera capaz de reunir en un solo vistazo su querer tomar una decisión y la desorganización subjetiva que le impide hacerlo cuando irrumpe el deseo de consumo, podría llegar a la conclusión de que requiere pedir ayuda para salir de la situación. Es decir, podría pensar que tiene que hacer un cambio significativo, pero que no está en condiciones de lograrlo por sí solo. El no llegar a esta conclusión es lo que podría explicar que finalmente sean los terceros significativos los que en la gran mayoría de los casos produzcan la consulta. La intervención de terceros permite la detención de la oscilación subjetiva, organizando un entendimiento de la situación y una búsqueda de ayuda que el sujeto no ha estado en condiciones de realizar, a pesar de haber sabido muchas veces lo mal que estaba.

Considerar estas dos características de la situación mental del sujeto antes de llegar a consultar, permite discutir la importancia de determinadas intervenciones terapéuticas iniciales que pretendan tomar en cuenta el problema de la motivación a realizar un cambio. Se ha visto, a partir del relato de los sujetos, que la desorganización y oscilación subjetivas dificultan que el sujeto realice realmente un cambio en su consumo adictivo. Pero, a su vez, estas parecen estar muy influidas por este mismo consumo. Se produce así una situación paradójica, consistente en que la detención o disminución del consumo adictivo es un elemento muy importante para favorecer la detención o disminución del consumo adictivo. Es razonable pensar que la detención permita que la desorganización subjetiva disminuya, ya que la situación mental y el entorno tienden a ordenarse con esa detención; y permita que la oscilación subjetiva deje de ser tan intensa, ya que entonces la estructura motivacional del sujeto no se ve permanentemente quebrantada por la conducta de consumo. Esto llevaría a que la situación mental cambie haciendo que la motivación, ya existente, pudiera traducirse en conductas sostenibles en el tiempo. Es decir, el entender lo que está ocurriendo psíquicamente, principalmente como una situación coyuntural al consumo de sustancias y sus consecuencias, y no como producto de una estructura pre-mórbida, 
invitará a intervenir directamente sobre elementos que modifiquen las características de esa situación, en lugar de dirigir los esfuerzos a cambiar la supuesta falla en la estructura a la base. Por otro lado, tener en cuenta la profunda desorganización subjetiva del sujeto hará considerar seriamente la posibilidad de incorporar terceros significativos al proceso, que puedan darle estabilidad a la búsqueda de ayuda.

El cómo se entienda lo que está ocurriendo con el sujeto antes de consultar, entonces, será de gran relevancia para la discusión que se ha tenido, y se tiene, en torno a cuáles son los elementos centrales que debiera tener una intervención terapéutica en este campo: ¿motivar o confrontar?; ¿favorecer o no, la detención o disminución del consumo?; ¿modificar los rasgos de personalidad a la base de la conducta adictiva?; ¿fomentar la participación de terceros significativos?; ¿utilizar métodos como la hospitalización y medicación para lograr la abstinencia?; ¿considerar el fenómeno de la adicción como síntoma, enfermedad o ambivalencia?; finalmente, ¿trabajar desde una elección por realizarse, desde el presionar o desde el obligar?

\section{Conclusión}

A partir de este estudio se identifican dos características principales de la situación mental de los sujetos con consumo adictivo de sustancias, a las que se ha llamado desorganización subjetiva y oscilación subjetiva. La identificación y definición de estas características permite asumir que el sujeto en esas condiciones ya tiene una importante motivación para el cambio en relación a su conducta adictiva. Sin embargo, esta motivación no logra concretarse conductualmente de manera estable, debido a un estar psíquicamente interferido, emocional y cognitivamente, que ofrece la posibilidad para que, con la irrupción de los deseos de consumo, el conjunto de motivaciones previo del sujeto se desdibuje. Esto lleva a que el sujeto oscile entre un querer e incluso decidir hacer un cambio, y un decidir dejarse llevar por los deseos de consumo. El sujeto no logra reunir ambos polos de la oscilación en un solo vistazo que le permitiera llegar a 
la conclusión que, entonces, requiere solicitar ayuda para detenerla. Tanto la desorganización como la oscilación subjetivas aparecen directamente influidas por el deseo de consumo, el consumo y sus consecuencias. Esto genera la paradoja de que para lograr hacer un cambio, es necesario ya haberlo hecho. El sujeto está preso principalmente de una situación mental coyuntural al consumo y sus consecuencias, más que de una estructura psíquica previa. La identificación y análisis de estas características lleva a validar, inicialmente, el rol que puede jugar la detención o disminución del consumo en una intervención terapéutica, así como la participación de terceros significativos en el proceso.

Finalmente, es necesario recalcar que este es un estudio preliminar, que utiliza una metodología de análisis que permite profundizar en la percepción de los sujetos, pero que, a su vez, es limitado en sus posibilidades de generalización debido a lo pequeño de la muestra y a las dificultades propias de un análisis de este tipo.

\section{Referencias}

Alcohólicos Anónimos (1995). Archivo informativo de Alcohólicos Anónimos. Recuperado el 2 de enero de 2006 desde http://www.alcoholicsanonymous.org

Alonso, L. (1995). Sujeto y discurso: el lugar de la entrevista abierta en las prácticas de la sociología cualitativa. En J. Delgado \& J. Gutiérrez (Comps.), Métodos y técnicas cualitativas de investigación en ciencias sociales (pp. 225-240). Madrid: Síntesis.

Azrin, N.H. (1976). Improvements in the community-reinforcement approach to alcoholism. Behavior Research and Therapy, 14, 339-348.

Beck, A., Wright, F., Newman, C. \& Liese, B. (1999). Terapia cognitiva de las drogodependencias. Espańa: Editorial Paidós.

Berenson, D. (1976). A family approach to alcoholism. Psychiatric Opinion, 13(1), 33-38.

Burton, N. (2005). Finding the Lost Girls: Multiplicity and Dissociation in the Treatment of Addictions. Psychoanalytic Dialogues, 15(4), 587-613. 
Cunningham, J. \& Breslin, F. (2004). Only one in three people with alcohol abuse or dependence ever seek treatment. Addictive Behaviors, 29, 221-223.

García Ferrando, M., Ibáńez, J. \& Alvira, F. (1989). El análisis de la realidad social. Métodos y técnicas de investigación. Madrid: Alianza Editorial.

Ghia, R. (2000). Toxicomanías e instituciones. Acerca de un tratamiento posible. En A. Donghi \& L. Vázquez (Comps.), Adicciones, una clínica de la cultura y su malestar (pp. 269-278). Buenos Aires: JVE Ediciones.

Ibáñez, J. (1979). Más allá de la sociología. México: Siglo Veintiuno.

Ingelmo, J., Ramos, M., Méndez, J.A. \& González, E. (2000). El enfoque Modular-Transformacional de la Psicopatología: su aplicación al problema de la dependencia de drogas. Aperturas Psicoanalíticas, 5. Recuperado el 2 de enero de 2006 desde http://www.aperturas.org

Johnson, V. (1973). I'll quit tomorrow. New York: Harper \& Rowe.

Kameniecki, M. (2000). La clínica institucional en un centro de asistencia en drogadependencias. En A. Donghi \& L. Vázquez (Comps.), Adicciones, una clínica de la cultura y su malestar (pp. 259-268). Buenos Aires: JVE Ediciones.

Kreither, J. \& López, C. (2001). Tratamiento de adicciones: una forma de comenzar. Psykhe, 10(2), 11-22.

Landau, J., Stanton, D., Brinkman-Sull, D., Ikle, D., McCormick, D., Garrett, J., et al. (2004). Outcomes with the ARISE Approach to Engaging Reluctant Drug- and Alcohol-Dependent Individuals in Treatment. The American Journal of Drug and Alcohol Abuse, 30(4), 711-748.

López, C. (2006). La adicción a sustancias químicas: ¿puede ser efectivo un abordaje psicoanalítico? Psykhe, 15(1), 67-77.

López, C. (2009). La decisión de entrar a un tratamiento de adicciones: motivación propia e influencia de terceros. Terapia Psicológica, 27(1), 119-127.

Luongo, L. (1995). Modalidad de consumo, particularidad del goce. En E. S. Sinatra, D. Sillitti \& M. Tarrab (Comps.), Sujeto, goce y modernidad III (pp. 47-52). Buenos Aires: Atuel.

Mazzini, A. (1994). Durmiendo con el enemigo. En E. S. Sinatra, D. Sillitti, \& M. Tarrab (Comps.) Sujeto, goce y modernidad II (pp. 163167). Buenos Aires: Atuel.

McDougall, J. (1994). Teatros de la mente. Ilusión y verdad en el escenario psicoanalítico. (2a Ed.) España: Julián Yébenes. (Trabajo original publicado en 1982).

Miller, W. \& Meyers, R. (1999). The community-reinforcement approach. Alcohol, Research and Health, 23(2), 116-122. 
Miller, W. \& Rollnick, S. (1999). La entrevista motivacional. Espańa: Editorial Paidós.

Morse, R. \& Flavin, D. (1992). The definition of alcoholism. Journal of American Medical Association, 268, 1012-1014.

Prochaska, J. \& Di Clemente, C. (1984). The transtheoritical approach: Crossing traditional boundaries of therapy. Homewood, IL: Dow Jones/Irwin.

Rado, S. (1926). Los efectos psíquicos de los intoxicantes: un intento de desarrollar una teoría psicoanalítica de los deseos morbosos. En S. Rado, Psicoanálisis de la conducta (pp. 33-48). Buenos Aires: Paidós.

Rado, S. (1933). El psicoanálisis de la farmacotimia (afición a las drogas). En S. Rado, Psicoanálisis de la conducta (pp. 73-89). Buenos Aires: Paidós.

Rosenfeld, H. (1964). Psicopatología de la drogadicción y el alcoholismo. Una revisión crítica de la literatura psicoanalítica. En H. Rosenfeld, Estados Psicóticos (pp. 253-281). Buenos Aires: Ediciones Hormé.

Sachs, H. (1923). La génesis de las perversiones. Recuperado el 10 de marzo de 2006 desde http://www.psi.uba.ar

Salamone, L.D. \& Godoy, C. (1994). Un análisis alucinante. En E. S. Sinatra, D. Sillitti, \& M. Tarrab (Comps.), Sujeto, goce y modernidad II (pp. 91-95). Buenos Aires: Atuel.

Salamone. L.D. (1995). El toxicómano y el goce cínico. En E. S. Sinatra, D. Sillitti, \& M. Tarrab (Comps.), Sujeto, goce y modernidad (pp. 101-104). Buenos Aires: Atuel.

Santisteban, D., Suárez-Morales, L., Robbins, M. \& Szapocznik, J. (2006). Brief strategic family therapy: Lessons learned in efficacy research and challenges to blending research and practice. Family Process, 45, 259-271.

Share, D., McCrady, B., \& Epstein, E. (2004). Stage of change and decisional balance for women seeking alcohol treatment. Addictive Behaviors, 29, 525-535.

Sillitti, D. (2000a). La hora de la verdad. En E. S. Sinatra, D. Sillitti, \& M. Tarrab (Comps.), Más allá de las drogas (pp. 69-73). La Paz: Plural Editores.

Sillitti, D. (2000b). La droga, la ciencia y el padre. En E. S. Sinatra, D. Sillitti, \& M. Tarrab (Comps.), Más allá de las drogas (pp. 183-187). La Paz: Plural Editores.

Sillitti, D. (2000c). La droga como respuesta a la culpa. En E. S. Sinatra, D. Sillitti, \& M. Tarrab (Comps.), Más allá de las drogas (pp. 201206). La Paz: Plural Editores. 
Sinatra, E. (2000a). El empuje al olvido: tres nombres del goce. En E. S. Sinatra, D. Sillitti, \& M. Tarrab (Comps.), Más allá de las drogas (pp. 171-182). La Paz: Plural Editores.

Sinatra, E. (2000b). La función del alcohol. En E. S. Sinatra, D. Sillitti, \& M. Tarrab (Comps.), Más allá de las drogas (pp. 189-200). La Paz: Plural Editores.

Sobell, M.B. \& Sobell, L.C. (2006). Obstacles to the adoption of low risk drinking goals in the treatment of alcohol problems in the United States: A commentary. Addiction Research and Theory, 14(1), 9-24.

Szapocznik, J. \& Williams, R. (2000). Brief Strategic Family Therapy: Twenty-Five Years of Interplay among Theory, Research and Practice in Adolescent Behavior Problems and Drug Abuse. Clinical Child and Family Psychology Review, 3(2), 117-134.

Tarrab, M. (1994). La heroína. En E. S. Sinatra, D. Sillitti, \& M. Tarrab (Comps.), Sujeto, goce y modernidad II (pp. 153-162). Buenos Aires: Atuel.

Tarrab, M. (2000a). La droga como partenaire. En E. S. Sinatra, D. Sillitti, \& M. Tarrab (Comps.), Más allá de las drogas (pp. 165-169). La Paz: Plural Editores.

Tarrab, M. (2000b). Las salidas de la toxicomanía. En E. S. Sinatra, D. Sillitti, \& M. Tarrab (Comps.), Más allá de las drogas (pp. 145-156). La Paz: Plural Editores.

Thomas, E., Adams, K., Yoshioka, M. \& Ager, R. (1990). Unilateral relationship enhancement in the treatment of spouses for uncooperative alcohol abusers. American Journal of Family Therapy, 18, 334-344.

Wild, C., Cunningham, J. \& Ryan, R. (2006). Social pressure, coercion, and client engagement at treatment entry: A self-determination theory perspective. Addictive Behaviors, 31(10), 1858-1872.

Fecha de recepción: 13 de junio de 2011.

Fecha de aceptación: 14 de diciembre de 2011. 